Highly Energetic Physical Processes and

Mechanisms for Emission from Astrophysical Plasmas

IAU Symposium, Vol. 195, 2000

P. C. H. Martens, S. Tsuruta, and M. A. Weber, eds.

\title{
Statistical Properties of Kerr BH Flywheel Model of QSOs/AGNs
}

\author{
S. Nitta \\ National Astronomical Observatory Japan, 2-21-1 Osawa, Mitaka, \\ 181-8588 Japan
}

\begin{abstract}
The aim of this work is to demonstrate the properties of the magnetospheric model around Kerr black holes, so-called the "flywheel" (rotation powered) model. The fly-wheel engine of the $\mathrm{BH}$ accretion disk system is applied to the statistics of QSOs/AGNs. Nitta, Takahashi, \& Tomimatsu clarified the individual evolution of the Kerr black-hole fly-wheel engine, which is parameterized by black-hole mass, initial Kerr parameter, magnetic field near the horizon, and a dimensionless small parameter. We impose a statistical model for the initial mass function of an ensemble of black holes using the Press-Schechter formalism. With the help of additional assumptions, we can discuss the evolution of the luminosity function and the spatial number density (population) of QSOs/AGNs. The result explains well the decrease of very bright QSOs and decrease of population after $z \sim 2$.
\end{abstract}

\section{Introduction}

We discuss the evolution of QSO/AGN activities under the fly-wheel (rotation powered) model, which is one of the plausible models for the powerful engine of AGNs including a rotating, central black hole (Kerr BH). This fly-wheel engine might not be as familiar as the fuel engine (accretion powered engine), but it is very attractive because this model can explain the evolution and lifetime of AGN activities very naturally.

By solving the magnetospheric equilibrium around a $\mathrm{Kerr} \mathrm{BH}$, we clarify the individual evolution of the output power of a Kerr BH fly-wheel engine (Nitta, Takahashi, \& Tomimatsu 1991). We here consider the statistical properties of an ensemble of the Kerr BH fly-wheel engines as a model of QSOs/AGNs, and obtain the evolutionary scenario of the luminosity function (LF) and the population.

\section{Luminosity Function and Population of QSOs/AGNs}

To make a model of an ensemble of Kerr $\mathrm{BH}$ engines, we assume the following formation scenario of BHs: 1) the $\mathrm{BH}$ initial mass function (IMF) is the PressSchechter type derived from the Compton drag scenario; 2) all of the BHs are assumed to be formed almost simultaneously at the epoch $z \sim 200$ with 3) nearly 

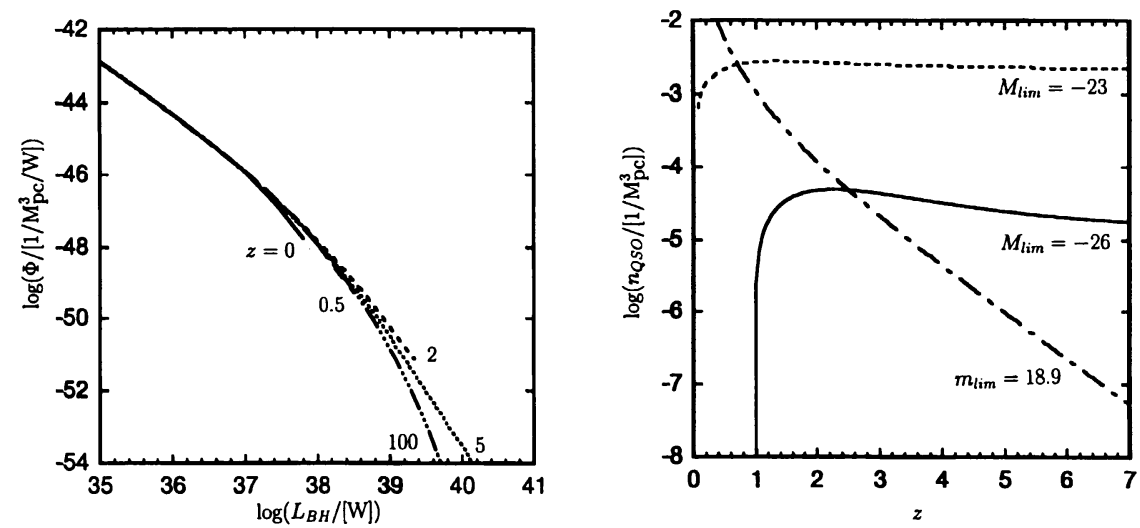

Figure 1. Evolution of luminosity function (left) and population (right.)

the maximum angular momentum; and 4) we assume the output power is equal to the bolometric luminosity (see Nitta 1999).

The typical result of the luminosity function is shown in Figure 1 (left). Moving from high to low $z$, the brighter end of the curve once lifts up for $5>z>2$, and then it drops and shrinks for $2>z>0$. We assume a detection limit $L_{\text {lim }}(t)$ of observation on the bolometric luminosity and count the number of BHs satisfying $L_{B H} \geq L_{l i m}$ as QSOs. (See Figure 1 (right). The absolute magnitude is $M \geq-26$ for the solid line and $M \geq-23$ for the dashed line. The dot-dash line denotes the population of QSOs with the apparent magnitude $m \geq 18.9$.) The tendencies of these results are consistent with observations.

\section{Discussion}

The main results argued here are obtained by integration of the individual evolution of fly-wheel engines over an ensemble of Kerr BHs denoted by the PressSchechter type IMF. We should note that this individual evolution is not a speculation but the result based on the MHD picture. We have tried to join the intrinsic Kerr BH magnetospheric physics, i.e., the fly-wheel model, with observational facts, and we have succeeded in presenting a mechanical model of the evolution, at least qualitatively.

\section{References}

Nitta, S. 1999, MNRAS, 308, 995

Nitta, S., Takahashi, M., \& Tomimatsu, A. 1991, Phys. Rev. D, 44, 2295 